\title{
Modelos Biomatemáticos com Retardamento: Métodos Numéricos
}

A.P. WYSE, M. RAFIKOV ${ }^{2}$, DEFEM - Departamento de Física, Estatística e Matemática, UNIJUÍ - Universidade Regional do Noroeste do Estado do RS, 98700-000 Ijuí, RS, Brasil.

Resumo. Existem processos e sistemas na natureza que devem ser modelados por equações diferenciais com retardamento. Uma grande dificuldade em relação a estas equações diz respeito à sua integração, pois métodos analíticos só são possíveis em casos muito particulares. Já os métodos numéricos, além de não poderem ser aplicados diretamente, precisam superar um problema de descontinuidade da primeira derivada no ponto inicial da integração.

\section{Introdução}

Muitos dos fenômenos encontrados na natureza precisam ser modelados por equações diferenciais com retardamento como, por exemplo, o crescimento de populações. Neste caso, a densidade populacional não responde imediatamente a certos estímulos, mas o faz após um período denominado "atraso" ou "retardamento".

Uma equação diferencial com retardamento apresenta-se da seguinte forma:

$$
\begin{gathered}
\frac{d x}{d t}=f(t, x(t), x(t-\tau)), \quad \tau>0 \\
x\left[t_{0}-\tau, t_{0}\right]=\varphi_{0}(t) .
\end{gathered}
$$

Aqui, o problema inicial é encontrar uma solução contínua $x(t)$ da equação (1.1) para $t>t_{0}$ sujeita à condição $(1.2)$, onde $\varphi_{0}(t)$ é uma função contínua conhecida e $t_{0}$ é o ponto inicial.

\section{Métodos de Integração Numérica}

Um dos métodos de resolução do problema (1.1) - (1.2) é o Método dos Passos, também conhecido como Método de Integração Sucessiva. Esse método consiste em

\footnotetext{
${ }^{1}$ Mestranda em Matemática

${ }^{2}$ Orientador de dissertação
} 
dividir o período de integração $\left[t_{0}, T\right]$ em intervalos de tamanho $\tau$ e substituir o termo com retardamento pela solução obtida no intervalo anterior. Portanto, devese encontrar a solução $x(t)$ do problema (1.1) - (1.2) da equação sem retardamento:

$$
\begin{gathered}
\frac{d x}{d t}=f\left(t, x(t), \varphi_{0}(t-\tau)\right) \\
t_{0} \leq t \leq t_{0}+\tau, \quad x\left(t_{0}\right)=\varphi_{0}\left(t_{0}\right) .
\end{gathered}
$$

Assim, o argumento $(t-\tau)$ fica no conjunto inicial $\left[t_{0}-\tau, t_{0}\right]$ e, conseqüentemente, o $3^{o}$ argumento $x(t-\tau)$ da função $f$ é igual à função inicial $\varphi_{0}(t-\tau)$.

Supondo que exista a solução $x(t)=\varphi_{1}(t)$ do problema inicial no intervalo $\left[t_{0}, t_{0}+\tau\right]$, por analogia obtém-se

$$
\frac{d x}{d t}=f\left(t, x(t), \varphi_{1}(t-\tau)\right)
$$

para $t_{0}+\tau \leq t \leq t_{0}+2 \tau, \quad x\left(t_{0}+\tau\right)=\varphi_{1}\left(t_{0}+\tau\right)$

$$
\frac{d x}{d t}=f\left(t, x(t), \varphi_{n}(t-\tau)\right)
$$

para $t_{0}+n \tau \leq t \leq t_{0}+(n+1) \tau, \quad x\left(t_{0}+n \tau\right)=\varphi_{n}\left(t_{0}+n \tau\right)$.

Porém, equações diferenciais com retardamento podem ser integradas analiticamente somente em casos excepcionais, ou seja, casos em que existam soluções analíticas em cada passo e que o retardamento $\tau$ não seja pequeno em relação ao intervalo em que se precisa encontrar a solução.

Mesmo assim, a idéia principal do Método dos Passos tem muita importância, pois os métodos numéricos habituais não podem ser aplicados diretamente à resolução de equações diferenciais com retardamento. Assim, a idéia do Método dos Passos deve ser embutida nos métodos numéricos.

Os métodos numéricos utilizados no presente texto são os métodos de quarta ordem de Runge-Kutta, Milne e Adams.

Uma das dificuldades encontradas na resolução de equações diferenciais com retardamento por métodos numéricos é a existência de um problema de descontinuidade para a $(\mathrm{k}+1)$-ésima derivada no ponto $t_{0}+k \tau$.

Realmente, no ponto $t_{0}$, embora seja satisfeita a condição $x\left(t_{0}\right)=\varphi_{0}\left(t_{0}\right)$, a primeira derivada $d x / d t$ é descontínua, pois

$$
\frac{d x}{d t}\left(t_{0}+\epsilon\right) \neq \frac{d \varphi_{0}}{d t}\left(t_{0}-\epsilon\right)
$$


onde $\epsilon$ é um infinitésimo. Somente num caso especial é possível obter uma continuidade da derivada da solução no ponto $t_{0}$.

No ponto $t_{0}+\tau$, a primeira derivada é uma função contínua, pois o lado direito da equação

$$
\frac{d x}{d t}=f(t, x(t), x(t-\tau))
$$

é função contínua em relação a $t$ no ponto $t_{0}+\tau$ desde que $x(t)$ é contínua no ponto $t_{0}$. Mas, a segunda derivada

$$
\frac{d^{2} x}{d t^{2}}=\frac{\partial f}{\partial t}+\frac{\partial f}{\partial} x(t) \frac{d x}{d t}+\frac{\partial f}{\partial x(t-\tau)} \frac{d x}{d t}(t-\tau),
$$

no ponto $t_{0}+\tau$, é descontínua, pois $\frac{d x}{d t}(t-\tau)$, quando $t=t_{0}+\tau$, é igual a $\frac{d x}{d t}\left(t_{0}\right)$ que, conforme dito anteriormente, é descontínua.

Utilizando o mesmo raciocínio, percebe-se que no ponto $t_{0}+2 \tau$ a derivada $\frac{d^{2} x}{d t^{2}}$ é contínua, logo que $\frac{d x}{d t}(t-\tau)$ e $x(t-\tau)$ são contínuas no ponto $t=t_{0}+2 \tau$, mas $\frac{d^{3} x}{d t^{3}}$ é descontínua, pois $\frac{d^{2} x}{d t^{2}}(t-\tau)$, no ponto $t_{0}+2 \tau$, é igual a $\frac{d^{2} x}{d t^{2}}\left(t_{0}+\tau\right)$, que é descontínua, como foi mostrado anteriormente.

Continuando esse raciocínio, observa-se então que no ponto $t_{0}+k \tau$, a $(k+1)$ ésima derivada é descontínua e as derivadas de ordem menor do que $k+1$ são contínuas nesse ponto.

Devido a esses pontos com derivadas descontínuas, os métodos numéricos ficam sujeitos a alguns problemas, principalmente os métodos explícitos.

\section{Simulações Numéricas}

Nas simulações que foram realizadas é possível observar que podem haver problemas quando utilizam-se os métodos numéricos sem tomar alguns cuidados importantes com relação aos problemas expostos na seç̧ão 2 .

Os modelos biomatemáticos utilizados foram os seguites: 
- Hutchinson

$$
\begin{aligned}
& \frac{d H(t)}{d t}=r H(t)\left[1-\frac{H(t-\tau)}{K}\right]-\alpha H(t) P(t) \\
& \frac{d P(t)}{d t}=-b P(t)+\beta P(t) H(t) \\
& \text { onde: } \\
& H=\text { densidade populacional de presas } \\
& P=\text { densidade populacional de predadores } \\
& r=\text { taxa de crescimento das presas } \\
& \tau=\text { tempo que as presas precisam para regenerarem-se do efeito causado pelo } \\
& \text { predador } \\
& K=\text { nível de saturação } \\
& b=\text { taxa de mortalidade dos predadores } \\
& \alpha \text { e } \beta=\text { coeficientes de iteração }
\end{aligned}
$$

- Hasting

$$
\begin{aligned}
& \frac{d H(t)}{d t}=r H(t-\tau)-d H(t)-f(H(t)) P(t) \\
& \frac{d P(t)}{d t}=P(t) f(H(t))-P(t)
\end{aligned}
$$

onde:

$\tau=$ período de imaturidade das presas

$r=$ taxa de natalidade das presas

$d=$ taxa de mortalidade das presas

$P(t) f(H(t))=$ efeito da predação

Os demais coeficientes têm a mesma definição anterior.

- Freedman e Rao

$$
\begin{aligned}
& \frac{d H(t)}{d t}=r H(t)-d H(t)-f(H(t)) P(t) \\
& \frac{d P(t)}{d t}=P(t) f(H(t-\tau))-P(t)
\end{aligned}
$$

onde:

$\tau=$ resposta do predador à predação

Os demais coeficientes têm a mesma definição anterior.

As Figuras 1 e 3 foram ampliadas em um mesmo intervalo a fim de ilustrar o problema percebido quando utilizou-se o método de Milne com valores iniciais calculados com o método Runge-Kutta. Nota-se ainda que o problema foi contornado 


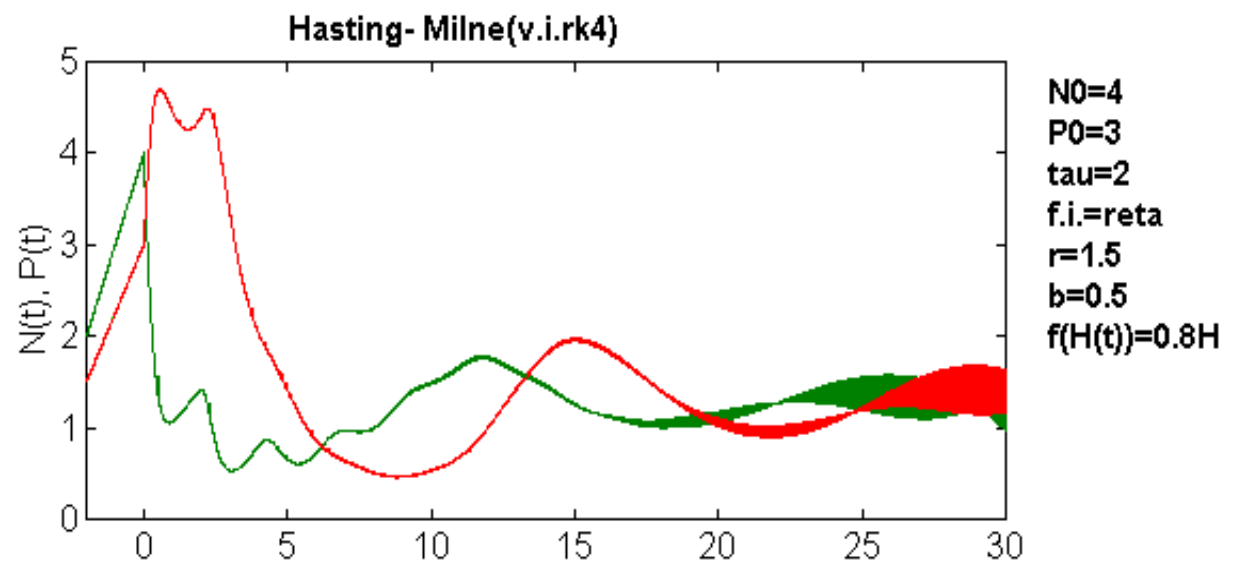

Figura 1: Simulação do modelo Hasting realizada com o método de Milne com valores iniciais calculados utilizando o método Runge-Kutta de quarta ordem. Nenhum tratamento especial foi dado aos pontos de descontinuidade das derivadas.

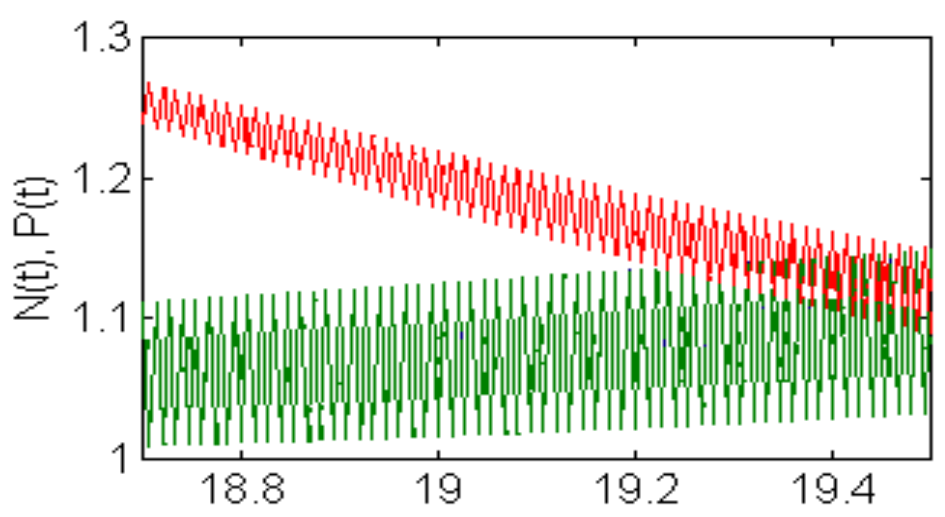

Figura 2: Ampliação de parte da Figura 1. 


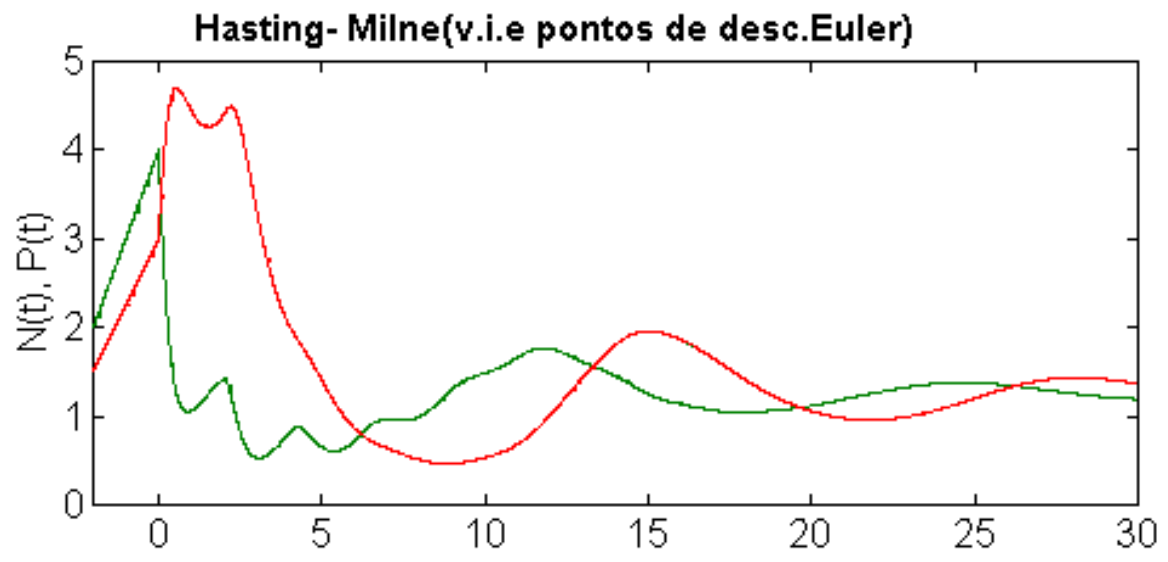

Figura 3: Simulação do modelo Hasting realizada com o método de Milne e com valores iniciais e pontos de descontinuidade calculados pelo método de Euler.

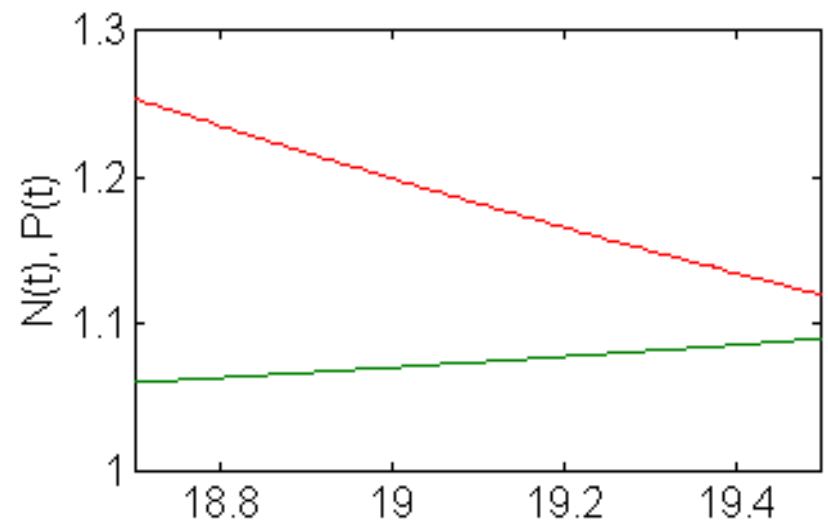

Figura 4: Ampliação de parte da Figura 3. 


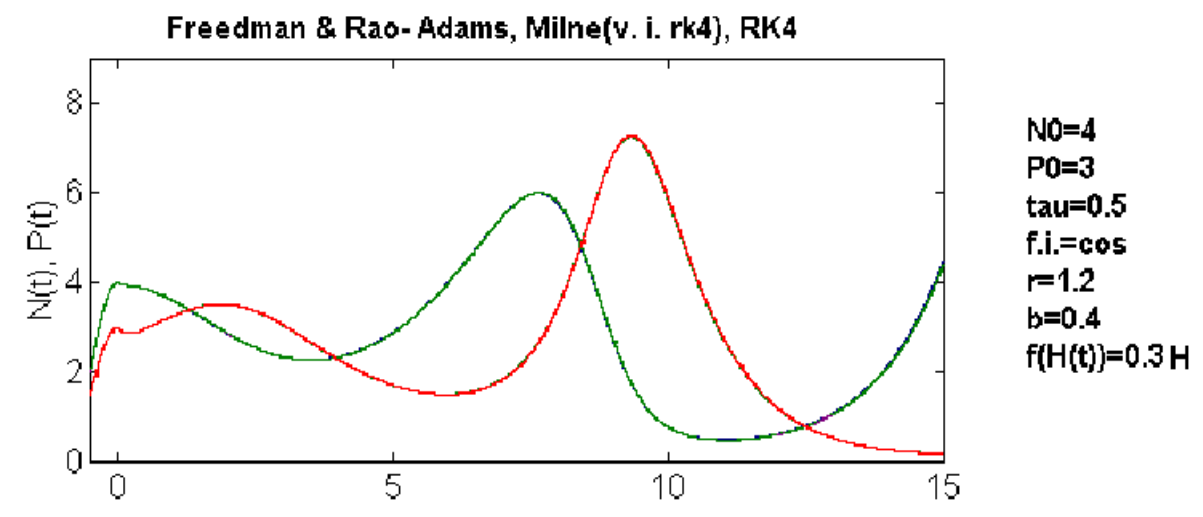

Figura 5: Simulação do modelo Freedman-Rao realizada com os métodos de Milne, Adams e Runge-Kutta; valores iniciais calculados pelo método de Runge-Kutta. Nenhum tratamento especial foi dado aos pontos de descontinuidade das derivadas.

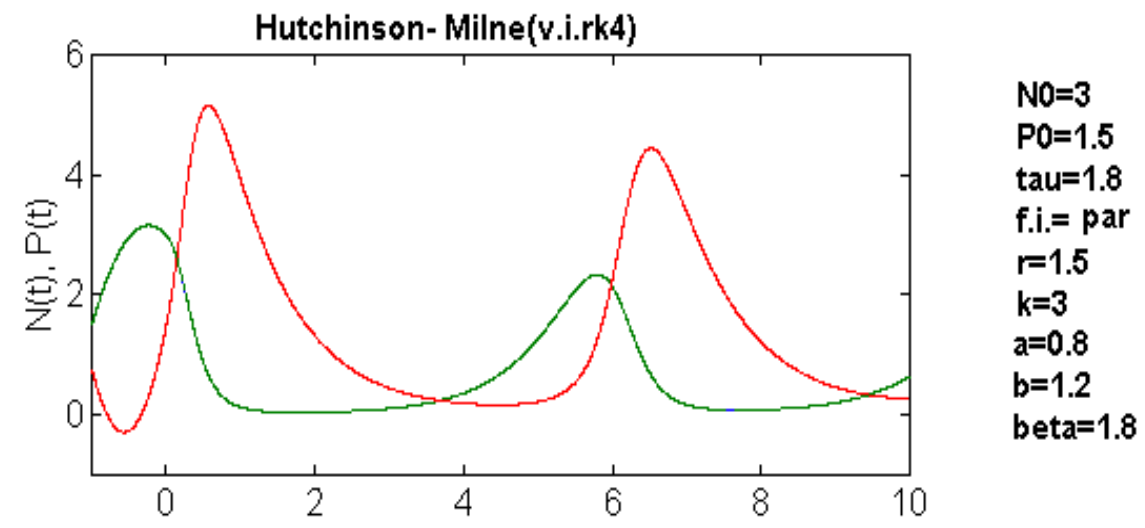

Figura 6: Simulação do modelo Hutchinson realizada com o método de Milne; valores iniciais calculados pelo método de Runge-Kutta. Nenhum tratamento especial foi dado aos pontos de descontinuidade das derivadas. 
quando utilizou-se o método de Euler para os valores iniciais e também nos pontos em que há descontinuidade em alguma ordem de derivada.

Na Figura 5, o método ilustrado é o de Milne, entre outros, visto que todos coincidiram. Apesar disso não surgiu nenhum tipo de anormalidade semelhante ao da Figura 1, que também ilustra Milne. A diferença é o modelo que está sendo integrado.

A Figura 6 ilustra um outro aspecto interessante do estudo realizado. Nota-se que não há descontinuidade da primeira derivada no ponto inicial $(t=0)$, pois foi escolhida uma função inicial especial de modo que houvesse continuidade da solução.

\section{Conclusão}

Como pôde ser visto ne secção anterior, realmente houve diferença entre alguns dos métodos numéricos utilizados. O método de Milne de quarta ordem foi o que apresentou problemas. Os métodos de Adams e Runge-Kutta, ambos de quarta ordem, mostraram um bom comportamento em relação à solução.

Uma sugestão para evitar o problema que surgiu na utilização do método de Milne (Figura 1), no caso em que a função inicial é inconveniente (ou seja, causa descontinuidade na primeira derivada no ponto inicial), seria a possibilidade de adotar o método de Euler para obter os primeiros valores necessários na utilização de métodos explícitos e, também, nos pontos de descontinuidade $t_{0}, t_{0}+\tau, \ldots, t_{0}+(n-1) \tau$, onde $n$ é a ordem do método numérico explícito utilizado.

\section{Referências}

[1] R. Bellman and K.L. Cooke, "Differential Difference Equations", New York: Academic Press, 1963.

[2] L.E. El'sgol'ts and S.B. Norkin, "Introduction to the theory and application of Differential equations with deviating arguments", New York: Academic Press, 1973.

[3] R.M. May, "Stability and Complexity in Model Ecosystems", New Jersey: Princeton University Press, 1974.

[4] R.M. May, Time-delay Versus Stability in Population Models with Two and Three Trophic levels. Ecology, 54 (1973), 314-325.

[5] H.I. Freedman and V.S.H Rao, The tradeoff between mutual interference and time lags in predator-prey systems.

[6] A. Hastings, Age dependent predation is not a simple process. Theor. Po. Biol., 23 (1983), 347-362. 